\title{
Review
}

\section{Transient receptor potential channel C5 in cancer chemoresistance}

\author{
Dong-xu $\mathrm{HE}^{1}$, Xin $\mathrm{MA}^{2, *}$ \\ ${ }^{1}$ National Engineering Laboratory for Cereal Fermentation Technology, Jiangnan University, Wuxi 214122, China; ${ }^{2}$ Department of \\ Cellular and Molecular Pharmacology, School of Medicine and Pharmaceutics, Jiangnan University, Wuxi 214122, China
}

\begin{abstract}
The transient receptor potential (TRP) superfamily contains at least 28 homologs in mammalian. These proteins form TRP channels are permeable to monovalent and divalent cations and participate in a variety of physiological functions. Dysregulation of TRP channels is responsible for numerous diseases. This review provides a brief short overview of mammalian TRP channels with a focus on TRPC5 and its role in cancers. Dysregulation of TRPC5 interrupts $\mathrm{Ca}^{2+}$ homeostasis in cancer cells, which activates signaling pathways that are highly associated with cancer progression, especially cancer chemoresistance. Based on the important role of TRPC5, we also discuss the potential of TRPC5 as a target for therapeutic intervention. Either direct targeting of TRPC5 or indirect interruption of TRPC5-related signaling pathways may effectively overcome cancer chemoresistance.
\end{abstract}

Keywords: transient receptor potential channel C5; cancer chemoresistance; drug target

Acta Pharmacologica Sinica (2016) 37: 19-24; doi: 10.1038/aps.2015.109; published online 14 Dec 2015

\section{Overview of TRP channels}

The 28 transient receptor potential (TRP) family members constitute 6 sub-groups in mammals: TRPC (canonical), TRPV (vanilloid), TRPM (melastatin), TRPP (polycystin), TRPML (mucolipin), and TRPA (ankyrin). Generally, TRP proteins reside in the plasma membrane as six transmembrane-domain polypeptide subunits, which usually require four subunits to assemble homo- or hetero-oligomeric pores into functional channels. TRP channels are permeable to both monovalent and divalent ions, except for TRPV5 and TRPV6, which are specific for $\mathrm{Ca}^{2+}$ ions, and TRPM4 and TRPM5, which are highly selective for monovalent cations.

TRP channels exist in almost every tissue and cell type. They provide a molecular framework for understanding important physiological functions, such as sensory transduction, neuronal growth-cone guidance, and vascular function. For example, their participation in the response to thermal and chemical nociceptive stimuli is one of the most widely-studied roles in sensory transduction. These stimuli activate TRPV1, TRPM8, and TRPA1 channels in afferent sensory neurons to induce pain ${ }^{[1]}$. In addition, TRP channels (especially TRPM5) are critical in taste receptors for sensing sweet, bitter, and

\footnotetext{
* To whom correspondence should be addressed.

E-mail maxin@jiangnan.edu.cn

Received 2015-09-05 Accepted 2015-11-01
}

umami modalities $^{[2]}$. Furthermore, TRPC channels participate in mediating attractive and repulsive growth-cone turning during axonal pathfinding ${ }^{[3]}$. TRP channels, such as those in the TRPC, TRPV, and TRPM subgroups, are also essential mediators of vascular functions such as arterial tone, angiogenesis, and permeability ${ }^{[4]}$.

Given the important physiological roles of TRP channels, TRP channelopathies are associated with a range of diseases ${ }^{[5]}$. For example, mutation of TRPP2 leads to autosomal dominant polycystic kidney disease ${ }^{[6]}$, and mutations in TRPV4 have been associated with skeletal dysplasias such as autosomal dominant skeletal dysplasia and Charcot-Marie-Tooth disease $^{[7,8]}$.

As one of the most widespread and life-threatening diseases, the relationship between cancer and TRP channels has also been studied. The main role of TRP channels in cancer is mediating a dysregulated $\mathrm{Ca}^{2+}$ homeostasis either by triggering $\mathrm{Ca}^{2+}$ entry pathways or changing membrane polarization. TRP channels interfere with critical cancer signaling pathways via dysfunction in $\mathrm{Ca}^{2+}$ signaling, causing perturbations in proliferation, apoptosis, gene transcription, and angiogenesis. For example, dysfunctional TRPM ${ }^{[9]}$, TRPV1 $1^{[10]}$, TRPC6, TRPC1, and TRPC4 ${ }^{[11]}$ promote malignancy, and TRPC6 contributes to the angiogenesis process in cancers ${ }^{[12,13]}$. Recently, we identified an essential role for TRPC5 in the occurrence and progression of chemoresistance in different cancers studied in 
vitro and in clinical samples ${ }^{[14-19]}$; this observation provides a new mechanism by which TRP channels modulate disease.

\section{Introduction to TRPC5}

TRPC5 is a homolog of the TRPC subgroup. The TRPC subgroup includes seven members (TRPC1-TRPC7). TRPC3, 6, and 7 are highly similar in structure and function, and TRPC4 and TRPC5 share structural and functional similarities. The TRPC proteins are ubiquitously expressed in mammalian cells, except for TRPC2, which is absent in humans, world monkeys, and apes ${ }^{[20,21]}$.

TRPC5 was originally identified in $1994^{[22]}$. In 1999, the human TRPC5 gene was successfully cloned from the region of Xq23, which encodes the protein with 99\% homology to mouse TRPC $5^{[23]}$. TRPC5 was first proposed to be a store-operated channel $^{[22]}$, but later studies also provided evidence of its receptor-operated properties ${ }^{[24,25]}$. The TRPC5 architecture remains poorly understood, but it is known to form a homo-oligomeric channel $^{[26]}$ and a hetero-oligomeric complex with TRPC1 and TRPC $4^{[27]}$. Accessory proteins also directly interact with TRPC5 during its activation, which is triggered by two controversial mechanisms with evidence supporting each ${ }^{[28]}$. In one of the hypotheses, TRPC5 is activated by a store-operated mechanism ${ }^{[29]}$ via direct interaction with $\mathrm{Ca}^{2+}$ sensor stromal interaction molecule 1 (STIM1) ${ }^{[30]}$. In the other proposal, agonistinduced activation of TRPC5 channels is store-independent but relies on the phospholipase $\mathrm{C}$ pathway ${ }^{[31,32]}$.

Like other TRP channels, TRPC5 activates $\mathrm{Ca}^{2+}$ pathways in response to physiological stimuli, including thioredoxin ${ }^{[33]}$, lanthanides ${ }^{[34]}$, nitric oxide ${ }^{[35]}$, lysophospholipids, growth factors $^{[36]}$, and stress. Increasing evidence has demonstrated that TRPC5 has a vascular function ${ }^{[37,38]}$ and is a cold sensor ${ }^{[26]}$. TRPC5 is particularly enriched in the brain and is tightly related in several neuronal processes, including growth-cone guidance $^{[39]}$, synaptic transmission ${ }^{[40]}$, neurite growth ${ }^{[41]}$, and fear-related behavior ${ }^{[42]}$.

Abnormal TRPC5 is known to contribute to several diseases. For example, infantile hypertrophic pyloric stenosis has been associated with single-nucleotide polymorphisms of the TRPC5 gene that disrupt smooth muscle control and lead to hypertrophy ${ }^{[43]}$. Additionally, TRPC5 dysfunction may cause occlusive vascular diseases by disrupting vascular smoothmuscle cells ${ }^{[44]}$. Moreover, focal segmental glomerulosclerosis is caused by unbalanced $\mathrm{Ca}^{2+}$ homeostasis through TRPC $5^{[45]}$.

\section{TRPC5 in chemoresistance}

Like other TRP channels, TRPC5 mutation that leads to interruption of $\mathrm{Ca}^{2+}$ homeostasis promotes cancer progression, and such dysfunction evokes a new pathway in the progression of cancer by developing chemoresistance.

Chemoresistance is one of the main features of malignancy; it allows cancer cells to become insensitive to chemotherapeutic agents and is the main cause of treatment failure. Overexpression of p-glycoprotein (p-gp) is the major attributor of chemoresistance; it actively pumps numerous foreign substances, including chemotherapeutic agents, out of cells ${ }^{[46]}$. The mecha- nism of $\mathrm{p}$-gp overexpression was unclear until TRPC5 was found to be overexpressed on the plasma membrane of $p$-gprich chemoresistant breast cancer cells ${ }^{[17]}$. Highly-expressed TRPC5 in human breast cancer cells can generate high $\mathrm{Ca}^{2+}$ channel activity, and the $\mathrm{Ca}^{2+}$ signal then activates the transcription factor NFATC3 (nuclear factor of activated T-cells, cytoplasmic 3) to initiate the transcription of p-gp, which finally induces overexpression of $\mathrm{p}$-gp and chemoresistance (Figure 1A). Furthermore, the TRPC5-NFATC3-p-gp pathway is activated in chemoresistant human colorectal cancer cells ${ }^{[47]}$, suggesting that cancer cells may commonly take advantage of this pathway to obtain high levels of p-gp. Given this assumption, the responses to various chemotherapeutic drugs could be hindered by TRPC5 overexpression due to the non-selective extrusion of amphipathic drugs by p-gp. These drugs include adriamycin, paclitaxel, and 5-fluorouracil, and each has a different mode of action.

$\mathrm{Ca}^{2+}$ influx is sufficiently versatile to transmit various cancer signals ${ }^{[48]}$. Cancer cells appear to employ TRPC5-related $\mathrm{Ca}^{2+}$ signals in addition to those producing $\mathrm{p}$-gp to acquire chemoresistance (Figure 1A). The Wnt/ $\beta$-catenin pathway is activated when $\mathrm{Ca}^{2+}$ is manipulated via TRPC5 channels ${ }^{[47]}$. Subsequently, Wnt/ $\beta$-catenin mediates the epithelial-mesenchymal transition of cancer cells, which is an important step in malignancy and chemoresistance development because of the high self-renewal, anti-apoptotic, and migratory features of mesenchymal cells ${ }^{[49]}$. Additionally, TRPC5/ $\mathrm{Ca}^{2+}$ dysregulation has been demonstrated to promote tumor angiogenesis mediated by hypoxia-inducible factor 1 (HIF-1 $)^{[50]}$. The dense vasculature in the tumor not only enables cancer cells to escape from sites exposed to high doses of anti-cancer drugs, but it also garners additional nutrition to aid tumor survival, which in turn results in a poor chemotherapeutic outcome.

How TRPC5 becomes enriched is still a mystery, although epigenetic regulation may play a role. MicroRNA-320a (miR$320 a)$, which may be a tumor suppressor ${ }^{[15,51,52]}$, has been demonstrated to target and degrade the mRNA of both TRPC5 and NFATC3. However, the promoter in the gene encoding miR$320 \mathrm{a}$ is hypermethylated in chemoresistant cancer cells, which silences the miRNA and activates the TRPC5-NFATC3-p-gp pathway ${ }^{[15]}$. Because epigenetic responses are sensitive to environmental stresses, as they can rapidly respond to certain circumstances (such as challenges from chemotherapeutic agents) to regulate various critical genes/networks, epigenetic regulation on TRPC5 and NFATC3 may suggest that this network is important and effective for developing chemoresistance.

Any tumor mass is heterogeneous, and some cancer cells may develop TRPC5-related chemoresistance when surviving chemotherapy while others may not directly confront the cytotoxicity of a drug and remain sensitive, which would not favor cancer progression. However, chemoresistant cancer cells usually adopt ways towards survival not only by inheriting genomic or epigenetic changes via replication but also by directly transmitting TRPC5 signals to their 'weaker' neighbors. To achieve transmission, the chemoresistant cancer cells pack the overexpressed TRPC5 proteins into mobile sub- 


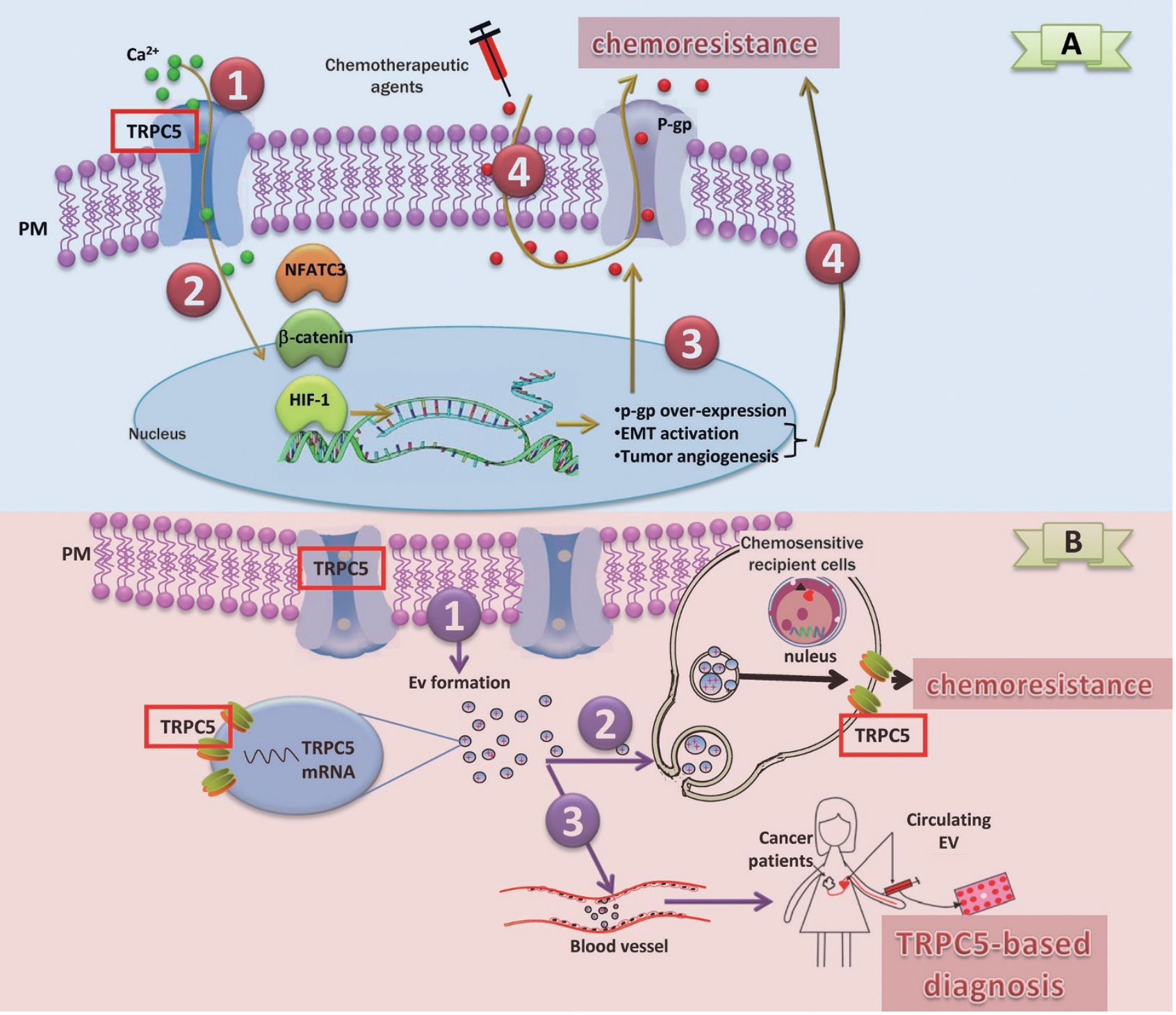

Figure 1. The mechanism of TRPC5 in mediating chemoresistance. (A) TRPC5 mediates the development of chemoresistance. In chemoresistant cancer cells, (1) overexpressed TRPC5 leads to the activation of the $\mathrm{Ca}^{2+}$ signaling pathway. (2) NFATC3, $\beta$-catenin, and HIF-1 are activated and lead to (3) p-gp overexpression, EMT pathway activation, and angiogenesis, respectively. (4) Overexpressed p-gp localizes onto the plasma membrane (PM), which can pump out chemotherapeutic drugs, and thus directly induces chemoresistance, EMT pathway activation, and angiogenesis and indirectly induces chemoresistance by modulating apoptosis, metastasis, and other pathways. (B) TRPC5 mediates the spreading of chemoresistance. In chemoresistant cancer cells that overexpress TRPC5, (1) Extracellular vesicles (EVs) are formed with the TRPC5 protein on the EV membrane and TRPC5 mRNA inside the EV. (2) The EVs are transferred to chemosensitive recipient cells, where TRPC5-mediated chemoresistance develops. (3) The EVs spread into the circulating system, and TRPC5 levels in the EV positively correlate with clinical level of chemoresistance. Therefore, TRPC5 levels in the EV represent a diagnostic marker for chemoresistance.

cellular structures known as extracellular vesicles (EVs) and transfer TRPC5 to sensitive cancer cells ${ }^{[18]}$ (Figure 1B). Because part of the phospholipid bilayer membrane of an EV contains the plasma membrane of the donor cells ${ }^{[53]}$, TRPC5 channels are already present on the EV membrane. When the EV finally merges with a recipient cell, TRPC5 is immediately activated on the plasma membrane that promotes the development of TPRC5-NFATC3-p-gp-mediated chemoresistance. Therefore, TRPC5's packaging into the limited EV volume serves a vital role in chemoresistant signaling in cancer cells. In addition, in the field of EV biology, the cumulative evidence suggests that ion channels, including TRPC5 and other different TRP channels, are preferentially built into EVs because they are easily transferred and can quickly activate a variety of signaling pathways in recipient cells. Therefore, it is valuable to explore the type and mechanism of TRP channels that are packaged and transferred by EV during certain physiological or pathological conditions.

To date, most chemoresistance is observed during the later period of treatment, where patients are suffering from toxic- 
ity in the presence of futile chemotherapy. However, TPRC5 is a good candidate for diagnosing chemoresistance and may help avoid exposing cancer patients to unnecessary chemotherapy. Based on our previous studies on cancer EVs, which are densely localized to the circulatory system and precisely reflect the pathological features of the donor cells ${ }^{[54-56]}$, we demonstrated that the quantity of TRPC5 mRNA in circulating EVs correlates with the clinical response to chemotherapy ${ }^{[18]}$ (Figure 1B). Therefore, if validated in a larger population, it may not only be possible to predict chemoresistance by analyzing TRPC5 levels in peripheral blood before initiating chemotherapy but also allow us to monitor the development of chemoresistance during or after chemotherapy.

\section{TRPC5 channel as a potential drug target}

To date, compared with other features of cancer progression, such as anti-apoptosis and angiogenesis, an effective means of inhibiting or reversing chemoresistance in the clinical setting remains elusive. However, TRPC5-based studies may provide insight into the development of anti-chemoresistance drugs. Inhibition of TRPC5 with siRNAs, the TRPC5-specific blocking antibody T5E3, and the pharmacological TRPC5 antagonist aminoethoxydiphenyl borate have been demonstrated to reduce chemoresistance to different chemotherapeutic agents in breast and colorectal cancers in vitro and in vivo. These observations suggest that therapeutic intervention targeting TRPC5 can effectively diminish chemoresistance. Additionally, combining TRPC5 modulators with chemotherapeutic agents may enhance routine chemotherapeutic regimes, as has been demonstrated in animal models ${ }^{[17]}$.

Because TRP channels, including TRPC5, occur in all cell types and modulate numerous cellular functions, side effects may present a major hurdle in TRP-based therapy. Therefore, TRP treatment accompanied by suitable drug delivery technology represents a reasonable strategy. In recent years, advances have been made in organ-specific drug delivery in the anti-tumor field, and nanocarriers with homing peptides could provide a basis for TRP-based treatment ${ }^{[57]}$. Furthermore, EVs have garnered excitement in the drug delivery field because they are naturally occurring stable vehicles that transport functional proteins, lipids, mRNAs, and miRNAs. Therefore, as TRP channels may vitally participate in the activity of EVs, and EVs naturally target specific recipient cells, engineered EVs may be quite effective for delivering agents in TRP-mediated therapies.

\section{Conclusion}

TRPC5 channel research has unveiled a pathogenic role for TRPC5 and highlights its potential for aiding the development of successful cancer therapies and diagnostic tools. Furthermore, translational research needs to be combined with clinical, pharmaceutical, and other fields of research to maximize the success of developing clinically useful TRPC5 drugs.

\section{Acknowledgements}

We thank Prof IC BRUCE for critical reading of the manu- script. This work was supported by the China National Natural Science Foundation 81572940 and 91439131 to Xin MA; 31200126 to Dong-xu HE; the Natural Science Foundation for Distinguished Young Scholars of Jiangsu Province (BK20140004 to Xin MA); the Program for New Century Excellent Talents in University of The Ministry of Education of China (NCET-12-0880 to Xin MA); the National High Technology Research and Development Program (863 Program) of China (SQ2015AA020948 to Xin MA); and Fundamental Research Funds for the Central Universities (JUSRP51311A and JUSRP51519 to Xin MA and Jin JIAN).

\section{References}

1 Julius D. TRP channels and pain. Annu Rev Cell Dev Biol 2013; 29: 355-84.

2 Ishimaru $\mathrm{Y}$ and Matsunami H. Transient receptor potential (TRP) channels and taste sensation. J Dent Res 2009; 88: 212-8.

3 Cui $K$ and Yuan $X$. TRP channels and axon pathfinding. In: Liedtke WB, Heller S, editors. TRP ion channel function in sensory transduction and cellular signaling cascades. Boca Raton (FL): CRC Press; 2007. Chapter 4

4 Earley S, Reading S, Brayden JE. Functional significance of transient receptor potential channels in vascular function. In: Liedtke WB, Heller S, editors. TRP ion channel function in sensory transduction and cellular signaling cascades. Boca Raton (FL): CRC Press; 2007. Chapter 26.

5 Nilius B, Owsianik G, Voets T, Peters JA. Transient receptor potential cation channels in disease. Physiol Rev 2007; 87: 165-217.

6 Qamar S, Vadivelu M, Sandford R. TRP channels and kidney disease: lessons from polycystic kidney disease. Biochem Soc Trans 2007; 35: 124-8.

7 Landoure G, Zdebik AA, Martinez TL, Burnett BG, Stanescu HC, Inada $\mathrm{H}$, et al. Mutations in TRPV4 cause Charcot-Marie-Tooth disease type 2C. Nat Genet 2010; 42: 170-4.

8 Rock MJ, Prenen J, Funari VA, Funari TL, Merriman B, Nelson SF, et al. Gain-of-function mutations in TRPV4 cause autosomal dominant brachyolmia. Nat Genet 2008; 40: 999-1003.

9 Thebault S, Lemonnier L, Bidaux G, Flourakis M, Bavencoffe A, Gordienko D, et al. Novel role of cold/menthol-sensitive transient receptor potential melastatine family member 8 (TRPM8) in the activation of store-operated channels in LNCaP human prostate cancer epithelial cells. J Biol Chem 2005; 280: 39423-35.

10 Lazzeri M, Vannucchi MG, Spinelli M, Bizzoco E, Beneforti P, Turini D, et al. Transient receptor potential vanilloid type 1 (TRPV1) expression changes from normal urothelium to transitional cell carcinoma of human bladder. Eur Urol 2005; 48: 691-8.

11 Thebault S, Flourakis M, Vanoverberghe K, Vandermoere F, Roudbaraki M, Lehen'kyi V, et al. Differential role of transient receptor potential channels in $\mathrm{Ca}^{2+}$ entry and proliferation of prostate cancer epithelial cells. Cancer Res 2006; 66: 2038-47.

12 Ge R, Tai Y, Sun Y, Zhou K, Yang S, Cheng T, et al. Critical role of TRPC6 channels in VEGF-mediated angiogenesis. Cancer Lett 2009; 283: 43-51.

13 Gkika D and Prevarskaya N. Molecular mechanisms of TRP regulation in tumor growth and metastasis. Biochim Biophys Acta 2009; 1793: 953-8.

14 Dong Y, Pan Q, Jiang L, Chen Z, Zhang F, Liu Y, et al. Tumor endothelial expression of P-glycoprotein upon microvesicular transfer of TrpC5 derived from adriamycin-resistant breast cancer cells. Biochem Biophys Res Commun 2014; 446: 85-90. 
15 He DX, Gu XT, Jiang L, Jin J, Ma X. A methylation-based regulatory network for microRNA 320a in chemoresistant breast cancer. Mol Pharmacol 2014; 86: 536-47.

16 Zhu Y, Pan Q, Meng H, Jiang Y, Mao A, Wang T, et al. Enhancement of vascular endothelial growth factor release in long-term drug-treated breast cancer via transient receptor potential channel 5- $\mathrm{Ca}^{2+}$-hypoxiainducible factor 1alpha pathway. Pharmacol Res 2015; 93: 36-42.

17 Ma X, Cai Y, He D, Zou C, Zhang P, Lo CY, et al. Transient receptor potential channel TRPC5 is essential for P-glycoprotein induction in drug-resistant cancer cells. Proc Natl Acad Sci U S A 2012; 109: 16282-7.

18 Ma X, Chen Z, Hua D, He D, Wang L, Zhang P, et al. Essential role for TrpC5-containing extracellular vesicles in breast cancer with chemotherapeutic resistance. Proc Natl Acad Sci U S A 2014; 111: 6389-94.

19 Wang T, Chen Z, Zhu Y, Pan Q, Liu Y, Qi X, et al. Inhibition of transient receptor potential channel 5 reverses 5 -Fluorouracil resistance in human colorectal cancer cells. J Biol Chem 2015; 290: 448-56.

20 Liman ER and Innan H. Relaxed selective pressure on an essential component of pheromone transduction in primate evolution. Proc Natl Acad Sci U S A 2003; 100: 3328-32.

21 Vazquez G, Wedel BJ, Aziz O, Trebak M, Putney JW. The mammalian TRPC cation channels. Biochim Biophys Acta 2004; 1742: 21-36.

22 Philipp S, Hambrecht J, Braslavski L, Schroth G, Freichel M, Murakami $M$, et al. A novel capacitative calcium entry channel expressed in excitable cells. EMBO J 1998; 17: 4274-82.

23 Sossey-Alaoui K, Lyon JA, Jones L, Abidi FE, Hartung AJ, Hane B, et al. Molecular cloning and characterization of TRPC5 (HTRP5), the human homologue of a mouse brain receptor-activated capacitative $\mathrm{Ca}^{2+}$ entry channel. Genomics 1999; 60: 330-40.

24 Schaefer M, Plant TD, Obukhov AG, Hofmann T, Gudermann T, Schultz G. Receptor-mediated regulation of the nonselective cation channels TRPC4 and TRPC5. J Biol Chem 2000; 275: 17517-26.

25 Okada T, Shimizu S, Wakamori M, Maeda A, Kurosaki T, Takada N, et al. Molecular cloning and functional characterization of a novel receptor-activated TRP $\mathrm{Ca}^{2+}$ channel from mouse brain. J Biol Chem 1998; 273: 10279-87.

26 Zimmermann K, Lennerz JK, Hein A, Link AS, Kaczmarek JS, Delling $\mathrm{M}$, et al. Transient receptor potential cation channel, subfamily C, member 5 (TRPC5) is a cold-transducer in the peripheral nervous system. Proc Natl Acad Sci U S A 2011; 108: 18114-9.

27 Strubing C, Krapivinsky G, Krapivinsky L, Clapham DE. TRPC1 and TRPC5 form a novel cation channel in mammalian brain. Neuron 2001; 29: 645-55.

28 Okada T, Shimizu S, Wakamori M, Maeda A, Kurosaki T, Takada N, et al. Molecular cloning and functional characterization of a novel receptor-activated TRP $\mathrm{Ca}^{2+}$ channel from mouse brain. J Biol Chem 1998; 273: 10279-87.

29 Kiselyov K, Xu X, Mozhayeva G, Kuo T, Pessah I, Mignery G, et al. Functional interaction between InsP3 receptors and store-operated Htrp3 channels. Nature 1998; 396: 478-82.

30 Yuan JP, Zeng W, Huang GN, Worley PF, Muallem S. STIM1 heteromultimerizes TRPC channels to determine their function as store-operated channels. Nat Cell Biol 2007; 9: 636-45.

31 Schaefer M, Plant TD, Obukhov AG, Hofmann T, Gudermann T, Schultz $G$, Receptor-mediated regulation of the nonselective cation channels TRPC4 and TRPC5. J Biol Chem 2000; 275: 17517-26.

32 DeHaven WI, Jones BF, Petranka JG, Smyth JT, Tomita T, Bird GS, et al. TRPC channels function independently of STIM1 and Orai1. J Physiol 2009; 587: 2275-98.

33 Xu SZ, Sukumar P, Zeng F, Li J, Jairaman A, English A, et al. TRPC channel activation by extracellular thioredoxin. Nature 2008; 451: 69-72.

34 Jung S, Muhle A, Schaefer M, Strotmann R, Schultz G, Plant TD. Lanthanides potentiate TRPC5 currents by an action at extracellular sites close to the pore mouth. J Biol Chem 2003; 278: 3562-71.

35 Yoshida T, Inoue R, Morii T, Takahashi N, Yamamoto S, Hara Y, et al. Nitric oxide activates TRP channels by cysteine S-nitrosylation. Nat Chem Biol 2006; 2: 596-607.

36 Flemming PK, Dedman AM, Xu SZ, Li J, Zeng F, Naylor J, et al. Sensing of lysophospholipids by TRPC5 calcium channel. J Biol Chem 2006; 281: 4977-82.

37 Xu SZ, Muraki K, Zeng F, Li J, Sukumar P, Shah S, et al. A sphingosine1-phosphate-activated calcium channel controlling vascular smooth muscle cell motility. Circ Res 2006; 98: 1381-9.

38 Xu SZ, Boulay G, Flemming R, Beech DJ. E3-targeted anti-TRPC5 antibody inhibits store-operated calcium entry in freshly isolated pial arterioles. Am J Physiol Heart Circ Physiol 2006; 291: H2653-9.

39 Kaczmarek JS, Riccio A, Clapham DE. Calpain cleaves and activates the TRPC5 channel to participate in semaphorin 3A-induced neuronal growth cone collapse. Proc Natl Acad Sci U S A 2012; 109: 7888-92.

40 Munsch T, Freichel M, Flockerzi V, Pape HC. Contribution of transient receptor potential channels to the control of GABA release from dendrites. Proc Natl Acad Sci U S A 2003; 100: 16065-70.

41 Hui H, McHugh D, Hannan M, Zeng F, Xu SZ, Khan SU, et al. Calciumsensing mechanism in TRPC5 channels contributing to retardation of neurite outgrowth. J Physiol 2006; 572: 165-72.

42 Riccio A, Li Y, Moon J, Kim KS, Smith KS, Rudolph U, et al. Essential role for TRPC5 in amygdala function and fear-related behavior. Cell 2009; 137: 761-72.

43 Everett KV, Chioza BA, Georgoula C, Reece A, Gardiner RM, Chung EM. Infantile hypertrophic pyloric stenosis: evaluation of three positional candidate genes, TRPC1, TRPC5 and TRPC6, by association analysis and re-sequencing. Hum Genet 2009; 126: 819-31.

44 Beech DJ. Ion channel switching and activation in smooth-muscle cells of occlusive vascular diseases. Biochem Soc Trans 2007; 35: 890-4.

45 Greka A and Mundel P. Balancing calcium signals through TRPC5 and TRPC6 in podocytes. J Am Soc Nephrol 2011; 22: 1969-80.

46 Bell DR, Gerlach JH, Kartner N, Buick RN, Ling V. Detection of P-glycoprotein in ovarian cancer: a molecular marker associated with multidrug resistance. J Clin Oncol 1985; 3: 311-5.

47 Wang T, Chen Z, Zhu Y, Pan Q, Liu Y, Qi X, et al. Inhibition of transient receptor potential channel 5 reverses 5-Fluorouracil resistance in human colorectal cancer cells. J Biol Chem 2015; 290: 448-56.

48 Monteith GR, McAndrew D, Faddy HM, Roberts-Thomson SJ. Calcium and cancer: targeting $\mathrm{Ca}^{2+}$ transport. Nat Rev Cancer 2007; 7: 51930.

49 Voulgari A and Pintzas A. Epithelial-mesenchymal transition in cancer metastasis: mechanisms, markers and strategies to overcome drug resistance in the clinic. Biochim Biophys Acta 2009; 1796: 75-90.

50 Zhu Y, Pan Q, Meng H, Jiang Y, Mao A, Wang T, et al. Enhancement of vascular endothelial growth factor release in long-term drug-treated breast cancer via transient receptor potential channel 5- $\mathrm{Ca}^{2+}$-hypoxiainducible factor 1alpha pathway. Pharmacol Res 2015; 93: 36-42.

51 Zhang Y, He X, Liu Y, Ye Y, Zhang H, He P, et al. microRNA-320a inhibits tumor invasion by targeting neuropilin 1 and is associated with liver metastasis in colorectal cancer. Oncol Rep 2012; 27: 68594.

52 Sun JY, Huang Y, Li JP, Zhang X, Wang L, Meng YL, et al. MicroRNA320a suppresses human colon cancer cell proliferation by directly targeting beta-catenin. Biochem Biophys Res Commun 2012; 420: 
787-92.

53 Raposo G and Stoorvogel W. Extracellular vesicles: exosomes, microvesicles, and friends. J Cell Biol 2013; 200: 373-83.

54 El AS, Lakhal S, Mager I, Wood MJ. Exosomes for targeted siRNA delivery across biological barriers. Adv Drug Deliv Rev 2013; 65: 391-7.

55 Muralidharan-Chari V, Clancy JW. Sedgwick A, D'Souza-Schorey C, Microvesicles: mediators of extracellular communication during cancer progression. J Cell Sci 2010; 123: 1603-11.

56 Skog J, Wurdinger T, van RS, Meijer DH, Gainche L, Sena-Esteves M, et al. Glioblastoma microvesicles transport RNA and proteins that promote tumour growth and provide diagnostic biomarkers. Nat Cell Biol 2008; 10: 1470-6.

57 Wang X, Li S, Shi Y, Chuan X, Li J, Zhong T, et al. The development of site-specific drug delivery nanocarriers based on receptor mediation. J Control Release 2014; 193: 139-53. 